A N N A L E S Annales de Bretagne et des Pays de l'Ouest

Anjou. Maine. Poitou-Charente. Touraine

109-4 | 2002

Les étrangers dans l'Ouest de la France (XVIII $-\mathrm{XXX}^{\mathrm{e}}$ siècle)

\title{
Des réfugiés politiques italiens en Maine-et-Loire : accueil et intégration (1845-1900)
}

Jérôme Hervé

\section{(2) OpenEdition}

Journals

Édition électronique

URL : http://journals.openedition.org/abpo/1512

DOI : $10.4000 /$ abpo.1512

ISBN : 978-2-7535-1488-1

ISSN : $2108-6443$

Éditeur

Presses universitaires de Rennes

Édition imprimée

Date de publication : 20 décembre 2002

Pagination : 79-95

ISBN : 978-2-86847-794-1

ISSN : 0399-0826

Référence électronique

Jérôme Hervé, « Des réfugiés politiques italiens en Maine-et-Loire : accueil et intégration

(1845-1900) », Annales de Bretagne et des Pays de l'Ouest [En ligne], 109-4 | 2002, mis en ligne le 20

décembre 2004, consulté le 21 avril 2019. URL : http://journals.openedition.org/abpo/1512 ; DOI :

10.4000/abpo. 1512 


\title{
Des réfugiés politiques italiens en Maine-et-Loire : accueil et intégration (1845-1900)
}

\author{
Jérôme HERVE \\ Étudiant en DEA d'histoire, Paris 1 - Panthéon Sorbonne
}

L'Ouest de la France n'a jamais été considéré comme une terre d'immigration. Pour les spécialistes de la question ${ }^{1}$, il semble que les sentiers balisés de l'exil ne conduisent que très rarement les migrants dans l'Ouest. Pour autant, cette région n'est pas une terra incognita, ni un espace totalement délaissé par les flux de population. En ce sens, la relative faiblesse de l'immigration, ne doit pas occulter l'originalité des expériences vécues par les étrangers venus s'installer dans ce "finistère " migratoire.

Le Maine-et-Loire, grâce à une tradition d'accueil maintes fois éprouvée, semble un département plus ouvert que les autres face à ces phénomènes. Les conséquences engendrées par les événements politiques en Italie, au milieu du XIX ${ }^{\mathrm{e}}$ siècle, vont permettre une rencontre bien improbable, entre des républicains romagnols, originaires des environs de Rimini et les habitants du Maine-et-Loire. L'histoire des Italiens à Saumur débute donc par l'arrivée surprise d'une cinquantaine de réfugiés au cours de l'année 1845. La prise en charge de ces hommes par la ville constitue en soi un premier enjeu. Quelles vont être, d'une part, les réactions des nouveaux arrivants face à ce changement forcé de domicile, et d'autre part, l'attitude des autorités et de la population face à cet afflux inattendu?

Ensuite, malgré le départ de la majorité des Romagnols quelques mois après leur arrivée, les quelques Italiens restés dans la ville seront à l'origine de la formation d'une micro-colonie italienne à Saumur. La question de l'insertion de ces étrangers dans la société d'accueil nourrit une seconde problématique, qui fera l'objet de la deuxième partie de cet exposé. Les stratégies d'insertion déployées par ces transalpins, les relations qu'ils ont pu entretenir avec les autochtones, leur participation à la vie locale, tant

1. Concernant cette question, il convient de se référer aux deux ouvrages suivants : ScHOR, Ralph, Histoire de l'immigration en France de la fin du XIXe siècle à nos jours, Paris, Armand Colin, 1996, 347 p. et Blanc-ChalEARD, Marie-Claude, Histoire de l'immigration, Paris, Repères, 2001, 120 p. 
au niveau économique que politique sont des indices qu'il faut exploiter afin de mieux cerner ce qu'a pu être leur vie à Saumur durant la période considérée.

\section{L'accueil des réfugiés romagnols à Saumur en 1845-1846}

Il convient tout d'abord de retracer rapidement le contexte politique italien de cette époque, ce qui permettra de comprendre les raisons qui ont poussé ces transalpins à prendre les armes et à se retrouver, malgré eux, dans le Maine-et-loire.

L'épisode français en Italie prend fin en 1815 par le congrès de Vienne qui consacre, sous le nom de Restauration, l'hégémonie massive de l'Autriche sur la péninsule. Les souverains en place replongent alors l'Italie dans un étroit conformisme. Cependant, les milieux intellectuels, acquis aux idées nationales et au libéralisme, cultivent le souvenir de la période française. Ainsi, cet élan de régénération (qui donnera plus tard le nom de Risorgimento au soulèvement italien) entretient une agitation endémique dans tout le pays. Les complots républicains naissent principalement en milieu urbain "à la tradition politique riche et ancienne ${ }^{2}$ ». En outre, ce sont plutôt les élites qui participent à ce mouvement. Les principaux événements se déroulent en Lombardie en 1833, puis à Bologne et surtout en 1844 en Calabre. Le coup de main des frères Banderia échoue, envoyant à la mort les dix-neuf membres qui composaient le commando. La France constitue la terre d'exil par excellence de ces républicains contestataires. Plusieurs vagues de fuorisciti se sont succédé dans l'hexagone de 1799 à 1845. Les Napolitains, les Piémontais, les Lombards et les Romagnols sont accueillis en France, principalement à Marseille et dans la capitale. Ainsi, les autorités françaises mettent en place une politique de prise en charge de ces nombreux exilés et permettent la diffusion des idées libérales portées par les Italiens. L'État subvient à leur besoin en leur allouant des subsides. En 1831, 1524 fuorisciti touchent un franc par jour à Paris ${ }^{3}$. En outre, certains personnages tels la princesse Belgiojoso et le marquis Arconati sont à la tête d'institutions philanthropiques. D'autres, comme Goberti, enseigne la philosophie. Tous les réfugiés ne s'installent pas dans ces deux villes. Ainsi, à la suite d'une rébellion survenue en Émilie-Romagne, les acteurs de cette insurrection n'ont pas d'autre choix que de venir se réfugier en France. Débarqués à Marseille, en provenance de Livourne, ils sont immédiatement dirigés vers une région éloignée du théâtre des opérations. Le Maine-et-Loire est donc choisi par l'administration, et plus précisément, la ville de Saumur. Ainsi, cette sous-préfecture va accueillir une cinquantaine de Romagnols sur les 312 qui ont été obligés de quitter l'Italie. Ils arrivent à destination à la fin de l'année 1845.

2. Guichonnet, Paul, Histoire de l'Italie, Paris, PUF, 1997, p. 79.

3. CRemieux, Benjamin, "L'émigration politique italienne en France sous la Monarchie de Juillet ", La société des études italiennes, Paris, 1936, p. 249-259, p. 250. 


\section{Qui sont-ils et d'où viennent-ils?}

Les cinquante-sept réfugiés Romagnols qui devaient théoriquement venir à Saumur, sont au nombre de cinquante-quatre, puisque deux d'entre eux vont tomber malades et rester à Aurillac, alors qu'un troisième s'est finalement embarqué pour l'Afrique afin de rejoindre la Légion étrangère française. Les autres arrivent en ordre groupé dans la commune au mois de novembre 1845. Ils sont tous originaires des environs de Rimini. Ainsi, les hommes qui résidaient à Faenza sont les plus nombreux (vingt), devant les habitants de Rimini (quatorze). Les autres vivaient dans des localités de taille moyenne telles que Bagnacavallo (six), Lugo (quatre), Russi (deux), Ancôme ou Pithigliani (un). Enfin, quelques-uns logeaient dans de petits villages comme Piano (un).

Tous les réfugiés politiques arrivés à Saumur sont des hommes. L'âge de la majorité d'entre eux oscille entre vingt-deux et vingt-sept ans, même si quelques-uns, plus vieux, font remonter la moyenne ( 27,8 ans). Le profil professionnel montre une population qui ne fait pas partie des couches les plus basses de la société italienne. En effet, l'artisanat domine (32). On dénombre, dans les métiers de l'habillement, neuf cordonniers, le même nombre de tailleurs et un chapelier. Les Romagnols figurent également dans le secteur de l'alimentation puisque trois boulangers et un charcutier sont installés à Saumur. Enfin, on peut recenser trois menuisiers, un lampiste, un verrier, un barbier ainsi que trois maçons. Huit autres transalpins sont commerçants : cinq négociants et trois cafetiers. Les métiers des services (trois domestiques), les professions libérales (un vétérinaire et un receveur du $12^{\mathrm{e} 4}$ ) ainsi que les métiers artistiques (un musicien) complètent ce tableau général. Enfin, neuf Romagnols sont recensés en tant que propriétaires.

La répartition sociologique de ces exilés confirme le profil des contestataires républicains. En effet, la plupart de ces hommes vivait en milieu urbain avant leur départ d'Italie. De plus, même s'ils ne font pas tous partie de l'élite des villes auxquelles ils appartiennent, il semble qu'une majorité d'entre eux possédait des biens et de la richesse. Malgré une position sociale assez élevée, la situation de ces réfugiés, lorsqu'ils arrivent à destination, est difficilement acceptable. Tout d'abord, il faut signaler que leur état sanitaire est bon. Seuls deux Italiens sont malades. Ainsi, " ces hommes sont tous valides, à l'exception du Sieur Toussaint Agneletti, momentanément malade à notre Hôtel-Dieu et du Sieur Louis Galanti, âgé de 47 ans, sans profession et déjà tout cassé ${ }^{5}$ ". Par contre, leur situation matérielle est catastrophique. Le maire de Saumur ${ }^{6}$ ne peut que le déplorer : " Les 45 réfugiés sont ici sans argent, sans pain, sans asile, sans linge, presque

4. Le $12^{\mathrm{e}}$ est un impôt versé par les habitants des villes en Émilie-Romagne.

5. Arch. dép. du Maine-et-Loire, $4 \mathrm{Z} 38$ : Lettre du maire de Saumur au préfet de Maineet-Loire, le 29 novembre 1845.

6. Charles Louvet est un riche banquier. Il est conseiller municipal en 1834 et maire de Saumur de 1844 à 1869. Il est également ministre à la fin du Second Empire. 
sans vêtement, en un mot dans le désœuvrement le plus absolu ${ }^{7}$. " Par conséquent, il incombe à la municipalité de fournir les premiers secours, de trouver un travail ainsi que de gérer la vie de ces Romagnols dans le Maine-et-Loire. Ces démarches ne vont pas s'effectuer sans poser de nombreux problèmes malgré la relative bienveillance de la population.

\section{Accueillir et faire vivre ces réfugiés dans le Saumurois}

L'un des problèmes à résoudre concerne l'aspect vestimentaire des réfugiés. La municipalité accorde aux Romagnols un cabaretier, une chemise coûtant 2,50 francs et une paire de sabots $(0,70 \text { franc })^{8}$. Ces hommes sont désormais habillés, il faut aussi les loger et les nourrir. Les réfugiés sont répartis sur quatre sites. Une majorité de transalpins mange sur leur lieu de vie. Enfin, il a fallu leur accorder quelques subsides pour leur permettre de vivre avant de trouver un emploi dans les environs. Ainsi, dans les premiers jours, la municipalité décide d'accorder 0,50 franc par jour et par réfugié. Ces premières mesures d'urgence n'ont pas vocation à perdurer très longtemps. En effet, le maire de Saumur est chargé, par le préfet, de trouver un emploi très rapidement à ces réfugiés : " Je vous prie de ne rien négliger pour leur procurer du travail, soit chez les ouvriers de la ville, soit sur le chemin de fer ${ }^{9}$."

La municipalité de Saumur eut beaucoup de mal à trouver un travail pour chaque réfugié. Ces difficultés reposent principalement sur un malentendu lié au choix de la ville. Il semble que ces Romagnols aient été dirigés sur Saumur en raison de la construction de la ligne de chemin de fer Paris-Nantes qui devait leur fournir du travail. Malheureusement, ces derniers ne souhaitent pas, compte tenu de leurs formations professionnelles, travailler sur ce chantier où les tâches exigées sont pénibles. Le maire de Saumur en est bien conscient lorsqu'il s'adresse au ministre de l'Intérieur : " Une partie de ces réfugiés appartient à des professions libérales, les autres exerçaient dans leur pays des métiers ou industrie qu'ils désirent continuer en France, tous manifestent la plus invincible répugnance pour les gros travaux manuels ${ }^{10}$. " En outre, "vu le trop grand nombre de ces réfugiés, accumulé dans une petite ville aussi petite que la nôtre, il est impossible de trouver des emplois libéraux ou industriels pour chacun ${ }^{11}$ ".

Trente d'entre eux vont réussir à trouver un poste à Saumur. Ils ont d'ailleurs souvent décroché un emploi dans le même secteur d'activité qu'en Italie. Par exemple, Mariano Ballanti et Benoît Rastelli ont pu repren-

7. Arch. dép. du Maine-et-Loire, $4 Z 38$ : Lettre du maire de Saumur au ministre de l'Intérieur, le 27 novembre 1845.

8. Arch. mun. Saumur, III147 : Fournitures adressées aux Romagnols, le 7. novembre 1845.

9. Arch. mun. Saumur, I II 147 : Lettre du préfet de Maine-et-Loire au maire de Saumur, le 22 novembre 1845.

10. Arch. dép. du Maine-et-Loire, $4 \mathrm{Z} 38$ : Lettre du maire de Saumur au ministre de l'Intérieur, le 27 novembre 1845.

11. Idem. 
dre leur métier de boulanger chez deux artisans : Dubois et Poitvin. Parfois, une légère reconversion est nécessaire. Ainsi, Antoine Baldi exerçait le métier de collecteur du $12^{\mathrm{e}}$ en Italie. Le maire de Saumur estime " qu'il paraît réunir les qualités propices à en faire un employé d'octroi. Il a 35 ans, bien portant, annonce de l'intelligence et semble être versé à ce genre de travail $^{12}$ ". Cependant, ces situations résolues ne concernent malheureusement pas tous les Romagnols. C'est ainsi que vingt transalpins sont envoyés dans sept localités du département ${ }^{13}$. Certains Italiens font preuve de courage en acceptant une place de terrassiers puisqu' " ils comprennent leur position et se plient donc à toute besogne ${ }^{14}$ ". Cependant, il ne s'agit pas d'une attitude généralisée. Dans la commune des Rosiers qui a reçu trois réfugiés, les autorités locales se plaignent de leur attitude. En effet, l'un d'entre eux a refusé une proposition d'emploi à Gennes comme casseur de pierres. Ainsi, "ils renoncent aux avantages que leur avait assurés monsieur le maire, en leur donnant le logement, le pain et la viande, attendu ont-ils dit qu'ils mourraient de faim avec cette ration et qu'ils préféraient revenir à leur dépôt ${ }^{15}$ ". Enfin, à l'image de Mathieu Bonazzali qui va trouver un emploi à Aurillac, certains exilés vont quitter le département.

L'État accorde des subsides aux Italiens qui sont au chômage. Cette somme est généralement de 0,75 franc par jour. Cependant, cette allocation est loin d'être suffisante pour vivre convenablement. Par conséquent, la solidarité saumuroise vient prendre le relais.

Deux manifestations sont mises sur pied au profit des Romagnols. Ainsi, Herman Bosco organise une représentation théâtrale. Ce dernier obtient la recette de 323,75 francs ${ }^{16}$. Cet argent va être consigné dans une cagnotte qui est répartie entre la fin du mois de novembre et le début de janvier 1846. La deuxième manifestation de soutien voit la venue d'un guitariste italien. M. Crema se propose de jouer quelques morceaux de musique avec la participation des musiciens et chanteurs locaux. Ainsi, soixante-dix personnes ont assisté au spectacle le 17 décembre 1845 pour une recette de 140 francs.

À côté de ces manifestations officielles, les dons des particuliers sont certes souvent moins importants, mais expriment peut-être plus encore la solidarité des habitants de la ville. La Société du commerce de Saumur va lancer une souscription qui permet de recueillir quatre-vingt-quinze francs. Ensuite, un don manuel de M. Dam permet de distribuer deux francs par réfugié. Enfin, le commissaire de police de la ville offre un franc aux Romagnols. La société de secours mutuel de Saumur vient aussi en aide

12. Arch. mun. Saumur, I II 147 : Lettre du maire de Saumur au directeur des contributions indirectes de Saumur, le 27 novembre 1845.

13. La ville de Baugé en reçoit notamment cinq, les communes de Longué et Beauforten-Vallée quatre.

14. Arch. mun. Saumur, I II 147 : Feuille de route pour les Romagnols se rendant à Longué, le 17 décembre 1845 .

15. Idem.

16. Arch. mun. Saumur, I II 147 : Représentation de Bosco Herman au profit des Romagnols, le 18 décembre 1845. 
aux réfugiés. Ainsi, elle a permis à sept Italiens de vivre ensemble en leur allouant 1,25 franc par jour. Les commerçants vont également faire preuve d'une belle solidarité. En effet, dans une conjoncture économique déprimée, les transalpins « font appel à la charité publique, principalement chez les fournisseurs de pain et de viande, qui leur font des avances, avec l'espoir d'être payés un jour par eux ${ }^{17}$ ".

Globalement, l'attitude de la population est positive par rapport à ces Romagnols. Aucune manifestation de réprobation, de mauvaise humeur n'a pu être retrouvée. De même, la prise en charge de ces réfugiés n'a eu, semble-t-il, aucune faille. Le dévouement des autorités locales est complet. La situation politique en Italie va permettre le déblocage de la situation.

\section{Quitter le Maine-et-Loire en bons termes}

Le 29 juillet 1846, les journaux saumurois annoncent une amnistie en faveur des Romagnols. Cette décision du pape Pie IX va faire réagir les Italiens. Ils se précipitent à Saumur " pour obtenir des passeports. Ils manifestent le plus vif intérêt de partir sans délai pour se rendre dans leur pays ${ }^{18}$ ". Le 8 août 1846, les réfugiés envoient une pétition au Cardinal Nonce de S.S Pie IX :

" Nous soussignés réfugiés romagnols en résidence à Saumur, avons l'honneur de vous exposer que nous désirons au plus vite rentrer dans notre patrie, comme fidèles sujets de S.S le pape Pie IX qui nous a ouvert généreusement les portes de cette patrie après laquelle nous gémissions. En conséquence, nous promettons que jamais nous ne prendrons les armes contre l'État ni contre la tranquillité publique et que nous saurons faire cas du généreux et loyal pardon que S.S nous accorde ${ }^{19}$."

Cependant, afin de quitter la France, les réfugiés ont besoin une nouvelle fois de l'aide saumuroise. Ils se rendent donc au conseil municipal pour se livrer à la déclaration suivante :

"Les réfugiés romagnols ont l'honneur de vous exposer, qu'au moment de partir pour leur patrie, ils se trouvent dans la nécessité d'avoir recours à votre bienveillante générosité, l'honneur leur commande de vous faire savoir que pour vivre, ils ont contracté des dettes dans leurs pensions et se voient dans l'impossibilité de pouvoir satisfaire leurs hôtes et ne peuvent se résoudre à quitter Saumur comme des gens mal famés, ils viennent avec une entière confiance se recommander aux dignes membres du conseil municipal de cette cité, qui n'a jamais fait défaut, envers des infortunés que des événements politiques ont poussé sur la terre hospitalière de la fameuse ville de Saumur ${ }^{20}$. "

17. Arch. dép. du Maine-et-Loire, $4 \mathrm{Z} 38$ : Lettre du maire de Saumur au sous-préfet de Saumur, le 22 janvier 1846.

18. Arch. mun. Saumur, I II 147 : Lettre du maire de Saumur au préfet du Maine-et-Loire, le 29 juillet 1846.

19. Arch. mun. Saumur, I II 147 : Pétition envoyée par les Romagnols au Cardinal Nonce de S.S Pie IX, le 8 août 1846.

20. Arch. mun. Saumur, I II 147 : Lettre des réfugiés romagnols au conseil municipal de Saumur, le $1^{\text {er }}$ septembre 1846. 
Une grande partie des réfugiés va quitter la ville dans les jours qui suivent cette déclaration. L'accueil des Romagnols a posé beaucoup de problèmes à la ville de Saumur. Leur nombre, leur profil professionnel, les paramètres économiques sont autant de difficultés qu'il a fallu surmonter. La relative docilité de ces hommes qui n'ont pas fait de prosélytisme politique dans le département et les réactions bienveillantes des autorités et des habitants ont permis un séjour relativement calme. Trois réfugiés décident de prolonger leur séjour dans le Maine-et-Loire malgré la deuxième amnistie accordée en septembre 1849. Ces derniers vont permettre la venue d'un certain nombre de leurs compatriotes à Saumur à la suite de cet épisode.

\section{Une intégration réussie?}

À partir du noyau initial issu de cette migration politique forcée, une petite colonie transalpine va se former et va perdurer jusqu'au début du $\mathrm{XIX}^{\mathrm{e}}$ siècle. La taille de cette implantation est réduite puisque le nombre de migrants va osciller entre vingt et trente membres. La faiblesse des courants migratoires produit généralement un effet d'invisibilité de la population concernée, tout cela dû à une absence d'engagement politique, syndical et associatif. Ainsi, les questionnements liés à la constitution de cette micro-colonie sont tributaires des caractéristiques de la zone d'accueil. En ce sens, plusieurs interrogations méritent d'être posées. Tout d'abord, l'immersion des Italiens dans un environnement peuplé en très grande majorité de Français est-elle un facteur accélérateur ou au contraire agit-elle comme un frein puissant à l'insertion? Ensuite, les vecteurs d'intégration mis à jour dans les régions de forte immigration fonctionnent-t-ils avec la même efficacité en Maine-et-Loire? Enfin, le mode d'implantation géographique des transalpins a-t-il pour conséquence une dissémination ou un regroupement des migrants dans la ville et permet-il l'existence d'une vie communautaire?

Face à l'apparente transparence des transalpins évoquée ci-dessus, il faut se doter d'indicateurs efficaces autorisant une analyse concrète de cette intégration. Cette tentative va s'opérer autour de trois axes principaux. L'insertion économique des Italiens, souvent mal délimitée, fera l'objet d'une présentation détaillée. Cela permettra, en entrant dans l'intimité socio-économique des migrants, de mesurer l'ampleur réelle de ce phénomène. Le deuxième facteur important concerne la participation politique des transalpins. Ce rapide aperçu va se cristalliser autour d'un personnage clé de cette micro-colonie, Angelo Bolognesi. Enfin, le recours à l'état civil se révèle indispensable pour éclairer le problème des solidarités migratoires dans un cadre $a$ priori peu favorable à une telle pratique.

\section{L'insertion économique des Italiens à Saumur}

On peut être frappé par la composition sociologique des migrants venus s'installer dans la ville de 1845 à 1900. En effet, ils font pratiquement 
tous partie de l'artisanat, quel que soit leur métier. Après une description rapide de ce phénomène, la reconstruction de quelques itinéraires professionnels s'avère nécessaire afin d'appréhender le contenu exact des patrimoines italiens.

L'artisanat transalpin à Saumur peut être divisé en trois catégories. La première regroupe les métiers de l'habillement. Les chapeliers occupent une place privilégiée en France au XIX siècle. Ainsi, " cette profession largement représentée à Marseille et à Lyon est surtout le privilège de la capitale $^{21}$ ". À Saumur, on dénombre au moins un chapelier à chaque recensement. Les ouvriers sont les plus nombreux. On peut citer, par exemple, le couple Molinar qui est employé par des commerçants français. Les patrons chapeliers sont plus rares puisque deux seuls exemples ont pu être relevés, notamment celui de la famille Liverani. Les métiers de la confection sont également une des professions de prédilection exercée par les transalpins. On peut noter la présence d'un patron tailleur nommé Jean Panzeri qui emploie deux compatriotes : Edouardo Sironi et Antonio Parazzoli. Quant à Antoine Frigério, après avoir été ouvrier chez un patron français, il devient propriétaire de son échoppe en 1901. La deuxième catégorie regroupe les emplois liés au travail des métaux. Une fois encore, différents corps de métiers sont représentés. Joseph Micheli, mouleur de son état, va se consacrer à son travail jusqu'à sa mort en 1894. Jean-Jacques Cottini, quant à lui, est ferblantier. Enfin, la dernière partie du contingent italien se consacre à une activité qui les place à la limite de l'artisanat et du grand commerce. En effet, à l'instar d'Angelo Bolognesi, plusieurs transalpins vont produire de la liqueur. Un certain nombre d'entre eux monte des affaires qui leur permettent d'accéder au négoce. Cette hiérarchisation des statuts distinguant les patrons et les ouvriers, les artisans des négociants implique sans doute une différenciation des conditions qu'il faut rendre moins abstraite.

La famille Liverani tient un commerce de chapellerie à Saumur de 1845 jusqu'au début du $\mathrm{xx}^{\mathrm{e}}$ siècle. Philippe Liverani est arrivé dans le département en novembre 1845 en compagnie des autres réfugiés romagnols. Il ouvre un magasin avant de faire appel à ses deux enfants, Joanna et Louis, en 1863. Arrivé à l'âge de la retraite, il leur cède son fond de commerce le 22 octobre 1874. Celui-ci est situé dans l'une des artères les plus commerçantes de la ville. Grâce à l'inventaire effectué lors de la cession du commerce, on peut noter la présence de quelques képis, signe que les officiers de l'école de cavalerie toute proche devaient s'approvisionner chez lui. Les enfants de l'Italien ont déboursé 6000 francs chacun ${ }^{22}$ pour occuper l'échoppe de leur père. La seule acquisition de cette famille est un terrain acheté par Louis Liverani pour la somme de 550 francs ${ }^{23}$. Lors de son décès,

21. MilzA, Pierre, Voyage en Ritalie, Paris, Plon, 1993, p. 207.

22. Arch. dép. du Maine-et-Loire, 5E 22/268 : Étude de Me Le Blaye, inventaire du commerce de chapellerie, le 22 octobre 1874.

23. Arch. dép. du Maine-et-Loire, 5E 22/268 : Étude de $\mathrm{M}^{\mathrm{e}}$ Le Blaye, achat d'un terrain, le 26 juillet 1883. 
ce dernier ne laisse aucun bien ${ }^{24}$, ce qui suggère des conditions de vie relativement modestes. Cette hypothèse est confirmée par le dossier de naturalisation de l'intéressé. Il est indiqué que le " postulant est sans fortune et vit de son petit commerce de chapellerie ${ }^{25}$ ". Joseph Micheli, un mouleur, partage cette précarité puisqu'il se retrouve sans aucune fortune lors de son décès ${ }^{26}$. L'autre représentant de ce corps de métier est Jean-Jacques Cottini. Les conditions de sa venue dans le Maine-et-Loire restent mystérieuses. En 1866, il se porte acquéreur d'une maison moyennant le paiement de 6155 francs $^{27}$. De plus, il devient propriétaire de l'hôtel d'Anjou deux ans plus tard ${ }^{28}$. En 1873, il cède son commerce à un Français. À la fin de sa vie, l'ancien ferblantier disposait de plus de 10000 francs $^{29}$.

Les commerçants de l'alimentaire ont également réussi à mettre sur pied quelques petites échoppes. Leur condition d'existence reste médiocre. Par exemple, Joachim Gionitti ne laisse que 97,75 francs ${ }^{30}$ à sa femme en 1866. En outre, il n'a acheté, ni vendu aucun bien. Le monde des liquoristes saumurois est fortement marqué par la présence transalpine. Angelo Bolognesi est le premier à avoir tenté sa chance dans ce secteur. Il est d'ailleurs associé durant quelques années à un certain Jean Combier, personnage sur lequel nous reviendrons ultérieurement. Cependant, après qu'une discorde soit intervenue entre les deux hommes, l'Italien va inventer une liqueur, "l'élixir Angelo ", qui va lui assurer un succès commercial conséquent.

À sa mort en 1879, le Romagnol possédait la somme de 131000 francs $^{31}$. En outre, il a effectué un grand nombre d'opérations financières. Son premier achat, une petite maison située à trois kilomètres de Saumur, date de 1854. Quelques années plus tard, entre 1864 et 1866, le liquoriste se porte acquéreur de plusieurs terrains à Dampierre, un village proche de la ville. Ces achats sont dictés par la nouvelle activité de l'Italien puisqu'il produit alors des vins champagnisés. Sa renommée et les retombées financières liées à son activité première lui ont permis de diversifier sa production. Enfin, de 1869 à 1873, le Romagnol devient propriétaire de biens immeubles de forte valeur, placés au centre de la ville. Il achète notamment l'hôtel

24. Arch. dép. du Maine-et-Loire, 3Q 14639 : Répertoire général, bureau de Saumur, le $1^{\text {er }}$ novembre 1909.

25. Arch. nat. BB ${ }^{11} 1670-3199 / \mathrm{X} 83$ : Dossier de naturalisation de Louis Liverani.

26. Arch. dép. du Maine-et-Loire, 3Q 14485 : Répertoire général, bureau de Saumur, le 5 janvier 1894.

27. Arch. dép. du Maine-et-Loire, 3Q 14594 : Répertoire général, bureau de Saumur, le 26 février 1866.

28. Arch. dép. du Maine-et-Loire, 3Q 14594 : Répertoire général, bureau de Saumur, le 23 janvier 1868 .

29. Arch. dép. du Maine-et-Loire, 3Q 14239 : Déclaration de mutation par décès, le 6 août 1891.

30. Arch. dép. du Maine-et-Loire, 3Q 14199 : Déclaration de mutation par décès, le 17 mars 1866.

31. Arch. dép. du Maine-et-Loire, 3Q 14222 : Registre des déclarations de mutations par décès, le 7 mai 1880. 
Boutet pour la somme de 28100 francs $^{32}$ au sous-préfet de Saumur. Cette augmentation du capital immobilier semblerait confirmer la position sociale dominante acquise par Angelo Bolognesi. Il devient membre du conseil municipal et du tribunal de commerce durant cette période. Les autres italiens qui ont travaillé dans la production de liqueur ont connu moins de réussite. Le frère d'Angelo, Mauro, est tout de même parvenu à amasser un petit patrimoine. En effet, il achète une maison rue Dacier (6642 francs $^{33}$ ) ainsi qu'une habitation de moindre valeur ${ }^{34}$. Auguste Christiani va être moins chanceux dans ses initiatives. À sa mort, il ne laisse aucun bien, ce qui ne veut pas dire qu'il a vécu dans la pauvreté. Cette absence provient de la dissolution d'une société ${ }^{35}$ de liqueur qu'il avait fondé avec le gendre d'Angelo Bolognesi, Charles Carichou, un ancien bijoutier reconverti. L'affaire ayant périclité, l'Italien a dû rembourser les nombreuses créances qu'il avait contractées avec son ancien associé. Cette situation n'est pas isolée puisqu'un autre transalpin, Ferdinand Pasquini, va subir les mêmes désagréments.

Quel bilan peut-on tirer de ces quelques histoires de vie? Tout d'abord, il semble que la mobilité sociale ascendante soit relativement modeste pour une majorité d'Italiens. La comparaison avec les sommes léguées par les habitants de Saumur dans les déclarations de mutation par décès montre que la population transalpine faisait plutôt partie des catégories humbles de la société. En effet, la plupart des Italiens laissent peu de biens à leur mort alors que dans les classes populaires, les montants moyens s'établissent à 1098 francs $^{36}$. Cependant, la brillante exception personnalisée par Angelo Bolognesi, et dans une moindre mesure par son frère Mauro et Jean-Jacques Cottini, montre que ce milieu permettait une ascension sociale conséquente. Ainsi, le ferblantier appartient à la classe moyenne ${ }^{37}$, tandis que le liquoriste s'est hissé au niveau des catégories aisées de la ville, puisque les classes supérieures léguaient 64033 francs $^{38}$, soit près de deux fois moins que le négociant italien. Ensuite, il convient de faire la distinction entre l'enrichissement personnel et la promotion sociale supposée. À l'image de Philippe Liverani, l'accession au patronat ne signifie pas forcément une amélioration des conditions d'existence. Enfin, la typologie et la localisation des placements italiens semblent indiquer un goût assez prononcé pour l'immobilier urbain. En tout cas, ceux qui avaient thésau-

32. Arch. dép. du Maine-et-Loire, 3Q 14608 : Répertoire général, bureau de Saumur, le 12 juillet 1869 .

33. Arch. dép. du Maine-et-Loire, 5E 42/319 : Étude de Me Mehouas, achat d'une maison, le 13 juin 1870 .

34. Arch. dép. du Maine-et-Loire, 3Q 14623 : Répertoire général, bureau de Saumur, le 3 septembre 1873 .

35. Arch. dép. du Maine-et-Loire, 3Q 14616 : Répertoire général, bureau de Saumur, le 24 mai 1885.

36. LANDAIS, Hubert, (dir.), Histoire de Saumur, Toulouse, Privat, 1997, p. 283.

37. La moyenne des sommes laissées par les membres des classes moyennes s'élève à 12339 francs, d'après LANDAIS, Hubert, op. cit., 1997, p. 283.

38. Idem. 
risé de l'argent l'ont dépensé, ce qui pourrait être le signe d'une volonté d'enracinement dans le territoire d'accueil. Les contours de l'intégration économique étant posés, il est temps d'aborder la participation politique des transalpins à Saumur.

\section{Contestataire ou conservateur?}

Comme on l'a suggéré dans l'introduction, Angelo Bolognesi est l'un des rares Italiens à s'être lancé en politique. La carrière du réfugié romagnol est particulièrement atypique puisque ses orientations sont très fluctuantes.

Angelo Bolognesi est arrivé à Saumur en 1845. Dès le début de son exil, il se lie d'amitié avec un certain Jean Combier, confiseur de son état. Ce dernier, " est considéré comme un des pères du parti républicain saumurois ${ }^{39}$ " et semble donc particulièrement sensible aux revendications portées par le Romagnol. Lors de la proclamation de la Seconde République en 1848, Angelo Bolognesi prend la parole en public. La scène se déroule sur la place de l'Hôtel de ville à l'occasion de la plantation d'un arbre de la liberté.

" Les Italiens, la plupart réfugiés politiques qui ont trouvé une généreuse hospitalité dans l'enceinte de cette ville, ont voulu, eux aussi, aujourd'hui profiter de cette solennelle et imposante cérémonie pour venir rendre hommage à la France de l'impulsion qu'elle a imprimé à tous les peuples de l'Europe et surtout à l'Italie qui n'attendait que son exemple pour prendre les armes contre les oppresseurs.

Peuple français, c'est sur vos traces que notre belle patrie a marché. De vous lui est venue la lumière, vous lui avez montré où est le bonheur des peuples. Elle aussi a voulu être libre comme vous et elle l'est. Désormais, vous pouvez tendre la main à l'Italien, il est votre égal, le joug qui l'opprimait ne l'humiliera plus.

Le peuple martyr a secoué ses chaînes. Pour toujours maintenant, le drapeau tricolore flottera là où naguère encore l'aigle impérial de l'Autriche insultait le sujet opprimé.

Le peuple qui demande à être votre frère en est digne, soyez en sûrs. Ils ont fait des prodiges de valeurs. À Milan, où devant chaque corps de garde, un canon était braqué sur le peuple, à Milan où du haut des remparts, les bouches à feu menaçaient la ville à être réduite en cendres au moindre mouvement, à Milan, où une garnison formidable tyrannisait le pauvre citoyen, à Milan, enfin, où les lâches barbares ont égorgé, impitoyablement les femmes et les vieillards et coupé les enfants en pièces pour les jeter par les fenêtres ou les porter en trophée au bout de leurs baïonnettes, à Milan, maintenant, on a vaincu, on est libre, libre et en république comme chez vous. Quelques jours encore et l'Italie entière sera régénérée. Aucune trace d'Autrichien n'y paraîtra si ce n'est celle du sang des innocents qu'ils ont égorgé et que la malédiction du ciel fera retentir sur eux ${ }^{40}$."

39. ÉMERIAu, Isabelle, Démocratie, Liberté, Patriotisme, le malentendu républicain, l'exemple du Maine-et-Loire (1848-1891), Rennes, Thèse de doctorat d'histoire, 1999, p. 471.

40. Le Précurseur de l'Ouest, 3 avril 1848. 
Cette contribution confirmant les positions libérales de l'exilé constitue la seule trace de l'engagement d'Angelo Bolognesi au côté des républicains. Celle-ci s'est déroulée dans une atmosphère particulière puisque le " printemps des peuples" exaltait les valeurs européennes et libérales. Cette absence provient-elle d'une lacune des sources, de la méfiance d'un étranger face à une activité qui pourrait lui valoir une expulsion ou d'un simple changement de convictions? Un début de réponse peut être apporté grâce à une lettre de dénonciation reçue par le commissaire de police de Saumur en 1858. Le sous-préfet avertit immédiatement son supérieur hiérarchique et lui expose le contenu de ce courrier ${ }^{41}$. Cette missive "signée " d'un employé de la police de Paris accuse ouvertement "les Sieurs Combier, Angelo et Joseph Bolognesi, tous trois liquoristes à Saumur, comme étant des conspirateurs et comme ayant fait partie du complot du 14 janvier $1858^{42}$ ". Le sous-préfet mentionne qu'ils adhèrent au parti républicain. Cependant, il attribue " à la malveillance les dénonciations dont ils font l'objet ${ }^{43}$ ". En effet, il estime " qu'autrefois ils étaient passablement avancés [mais] ils sont devenus très modérés depuis qu'ils ont acquis de la fortune ". Ainsi, " il ne serait pas impossible qu'un sentiment d'envie ou de vengeance ait dicté la lettre anonyme écrite contre $\operatorname{eux}^{44}$ ». Angelo Bolognesi entre au conseil municipal en 1871 sur la liste de la droite.

Cette carrière fait donc passer l'Italien du statut de réfugié contestataire à celui de conseiller municipal prônant des valeurs conservatrices. L'ascension sociale du Romagnol doit être mise en rapport avec l'évolution des conceptions politiques de cet homme. Il semble que cet engagement soit un cas isolé même si l'un de ses cousins, Joseph, a également eu des rapports avec le parti républicain. Toutefois, cet exemple est porteur de sens. En effet, comme dans les régions de forte immigration, le milieu d'accueil a permis l'intégration d'un étranger dans les plus hautes sphères politiques locales. Le dernier point concerne plus spécifiquement la sociabilité interne des transalpins.

\section{Une histoire vue de l'intérieur}

Il faut apercevoir les caractéristiques de l'implantation italienne à Saumur. Les transalpins habitent plutôt dans le centre ville. Cependant, on peut remarquer un éparpillement des migrants au sein de l'espace urbain. Il n'existe pas de " petite Italie " à Saumur.

41. Arch. dép. du Maine-et-Loire, 1M6/46 : Lettre du sous-préfet de Saumur au préfet du Maine-et-Loire, le 2 avril 1858.

42. Idem. L'auteur de la lettre fait référence à l'attentat d'Orsini. Ce Romagnol a tenté de tuer le couple impérial le soir du 14 janvier 1858 alors qu'il se rendait à l'opéra. Le terroriste espérait déclencher une révolution en France.

43. Idem.

44. Id. 
Cette ventilation large des Italiens a-t-elle pour corollaire un relâchement des liens entre les exilés? En l'absence de témoignages oraux et de sources explicites (presse italienne, créations d'associations diverses, etc.), il est difficile de rendre compte des relations qui ont pu exister entre ces migrants. Pourtant, l'utilisation de l'état-civil ${ }^{45}$, complétée par quelques documents notariés, permet de saisir certains aspects de cette vie en exil.

Il semble que la famille Bolognesi, et particulièrement l'un de ses membres, Joseph, cousin du contestataire républicain, soit très présente. Ainsi, il assiste au mariage ${ }^{46}$ entre Marie Liverani et Florent Jacob. Ces deux familles originaires de Russi, en Émilie-Romagne, entretenaient déjà des relations amicales dans leur pays d'origine. En effet, lors d'une opération financière effectuée par Louis Liverani, cousin de la mariée, qui avait pour objet l'achat d'une maison en Italie, les deux témoins Angelo Bolognesi et Joseph Bolognesi " ont déclaré bien connaître les dits époux Bartholo Liverani et Maria Patriani en Italie ${ }^{47}$ ". Joseph Bolognesi participe également au mariage ${ }^{48}$ entre Joachim Gionitti marchand de comestible, originaire de Castiglione et Perrine Demazière. Enfin, il est témoin lors du décès du fils de son cousin liquoriste, le nommé Sante Minghetti ${ }^{49}$. Angelo Bolognesi, quant à lui, est nommé mandataire par Philippe Liverani lorsqu'il cède son commerce à ses deux enfants ${ }^{50}$. En outre, rappelons que le négociant a prêté l'argent nécessaire à Auguste Christiani pour qu'il puisse débuter son activité de liquoriste ${ }^{51}$. Ce dernier est d'ailleurs l'un des quatre témoins du mariage qui unit Stéphane Pelletier et Ernesta Martelli ${ }^{52}$. Cette Italienne issue d'une famille originaire de Bologne est la fille de Louis Martelli ${ }^{53}$, marié avec une certaine Dominique Bolognesi.

Il semble difficile de tirer des conclusions générales de ces exemples isolés. Logiquement, il apparaît que c'est la famille Bolognesi qui sert de repère aux nouveaux arrivants. La réussite d'Angelo, l'ancienneté de son installation dans la ville les destinent tout particulièrement à servir d'intermédiaires et de médiateurs lors de leur séjour à Saumur.

45. Nous avons consulté les actes de naissances, mariages, décès des Italiens à Saumur. 46. Arch. dép. du Maine-et-Loire, 6E 328/135 : Mariage entre Marie Liverani et Florent Jacob, le 18 février 1884 .

47. Arch. dép. du Maine-et-Loire, 5E 22/260 : Procuration par Louis et Joanna Liverani à Sanzi.

48. Arch. dép. du Maine-et-Loire, 6E 328/135 : Mariage entre Joachim Gionitti et Perrine Demazière, le 14 mars 1890 .

49. Arch. dép. du Maine-et-Loire, 6E 328/138 : Décès de Sante Minguetti, le 23 novembre 1891.

50. Arch. dép. du Maine-et-Loire, 5E 22/268 : Cession de commerce, le 22 octobre 1874.

51. Arch. dép. du Maine-et-Loire, 5E 22/259: Vente par Angelo Bolognesi d'une cave à Auguste Christiani et Charles Carichou.

52. Arch. dép. du Maine-et-Loire, 5Mi : État civil de la ville de Saumur, mariage entre Stéphane Pelletier et Ernesta Martelli, le 26 août 1869.

53. Arch. dép. du Maine-et-Loire, 5Mi : État civil de la ville de Saumur, mariage entre Stéphane Pelletier et Ernesta Martelli, le 26 août 1869. 
Nous avons donc retrouvé douze actes impliquant un Italien dans lequel un ou plusieurs de ses compatriotes étaient témoins. Cela peut paraître peu $^{54}$. Toutefois, en comparaison du cas angevin (dix actes repérés durant la même période pour un contingent italien nettement supérieur ${ }^{55}$ ), il semblerait que les transalpins de Saumur entretiennent des relations plus étroites. Les causes de ce phénomène sont doubles. Tout d'abord, il semble que l'origine géographique des migrants ait joué un rôle prépondérant dans le maintien des contacts en France. En effet, le bassin migratoire est peu étendu puisque la plupart des transalpins proviennent des environs de Bologne. Ainsi, une micro filière d'immigration a vu le jour, prenant parfois même un caractère familial. En outre, le maintien des relations avec la région d'origine ainsi qu'un profil sociologique relativement proche renforcent encore les liens entre les immigrés. Le deuxième élément concerne la zone d'accueil. La taille de la ville peut également influer sur ces relations. Malgré la relative dispersion des transalpins, il était tout de même facile de se retrouver dans un espace peu étendu.

Le premier épisode de l'histoire commune entre les Italiens et la ville de Saumur s'est bien déroulé malgré les nombreuses difficultés rencontrées. L'accueil des Romagnols par la population et la municipalité a permis aux exilés de vivre convenablement malgré la précarité de leur situation. On aurait pu croire que le départ massif des réfugiés sonnerait le glas des relations entre les transalpins et le Maine-et-Loire. Il n'en fut rien.

La deuxième partie de l'exposé a donc porté sur le problème de la formation d'une micro-implantation italienne dans une zone marquée par une très faible présence étrangère. À l'aune de cette description, il est possible de tirer quelques enseignements de ce travail.

Premièrement, Saumur se prêtait bien à cette immigration artisanale car cette ville commerçante possédait un marché et une clientèle disponibles. La mobilité sociale ascendante des Italiens a été, sauf exception, relativement modeste. Ce phénomène n'est d'ailleurs pas une spécificité saumuroise puisque Pierre Milza a montré que l'ascension sociale transalpine était très limitée dans le reste de l'hexagone. Ainsi, "l'impression générale, enregistrée de manière empirique, va plutôt dans le sens d'une progression modeste ${ }^{56}$ ". Concernant la vitesse de l'insertion économique à Saumur, l'absence de point de comparaison rend difficile toute interprétation. Angelo Bolognesi a acheté sa première maison en 1854, soit une dizaine d'années après son arrivée dans le Maine-et-Loire. Son frère, Mauro,

54. Un des facteurs explicatifs est la grande instabilité de la population italienne en France. En effet, le nomadisme est l'une des caractéristiques majeures de l'immigration transalpine. À Saumur, le phénomène est un peu moins accentué qu'à Angers mais le renouvellement de population d'un recensement à l'autre ne facilite pas le maintien de relations interpersonnelles entre Italiens.

55. En 1872, on recense 44 Italiens à Angers alors qu'ils ne sont que 17 à Saumur.

56. MilzA, Pierre, op. cit., 1993, p. 373. 
et Jean-Jacques Cottini sont devenus propriétaires de biens immobiliers de valeur après quelques années de résidence dans la ville. Gérard Claude, dans sa thèse consacrée à l'intégration des Italiens et des Espagnols en Provence, indique que les premières acquisitions des colons transalpins " interviennent péniblement une trentaine d'années après l'installation définitive $^{57}$ ". De même, Philippe Rygiel, en étudiant l'insertion des étrangers dans le département du Cher pendant l'entre-deux-guerres note que " la mobilité sociale ascendante apparaît alors comme un phénomène rare, lent et de faible amplitude ${ }^{58}$ ". Toutefois, la différence des milieux d'accueil et des flux migratoires ${ }^{59}$ ne permet pas de conclure à une intégration plus rapide des Italiens à Saumur. Ainsi, le fait que le milieu d'accueil soit composé en très grande majorité d'autochtones ne constitue pas une barrière infranchissable à l'établissement économique de ces migrants même si la réussite sociale n'est l'apanage que d'une minorité d'individus.

L'entrée en politique constitue un facteur d'insertion à part entière. Le Maine-et-Loire est considéré comme un département acquis au conservatisme. Par conséquent, l'accueil de réfugiés républicains aurait pu poser de sérieux problèmes. C'était sans compter sur les particularismes politiques locaux. La ville de Saumur est l'un des bastions du républicanisme dans le département. Cette caractéristique a permis une intégration plus aisée d'Angelo Bolognesi et de sa famille.

La présence d'un entre-soi dans un tel espace a de quoi surprendre. Cependant, la mise en place d'un axe privilégié de migration entre l'ÉmilieRomagne et le Maine-et-Loire peut expliquer le maintien de ces contacts. Cela n'est pas sans conséquence sur l'insertion italienne. En effet, dans les régions de forte immigration, la formation de " petites Italies " favorisait une première phase d'intégration en douceur du migrant. Un tel milieu n'existe pas à Saumur. Malgré cela, la relative " cohésion " du contingent évite aux nouveaux arrivants l'isolement complet et permet peut-être d'amortir le choc de la transplantation. Ainsi, le triptyque d'insertion désormais bien connu mêlant les facteurs politiques, économiques et sociaux semble avoir fonctionné à deux vitesses. Pour une majorité d'Italiens, ce processus a tourné au ralenti et ne leur a pas permis de s'enrichir beaucoup. Pour les autres, ceux qui ont réussi, ce milieu d'accueil constitue un terreau favorable pour une intégration rapide et une ascension sociale élevée.

57. CLAUDE, Gérard, Les étrangers en milieu rural : un siècle d'immigration italienne et espagnole en Provence de 1850-1940, Thèse de doctorat d'histoire, Aix-en-Provence, 1992, 2 volumes.

58. RYGIEL, Philippe, Mais où sont les immigrés d'Antan, trajectoires sociogéographiques de familles issues de l'immigration européenne implantées dans le Cher durant l'entre-deuxguerres, Thèse de doctorat d'histoire, Besançon, 1996, 3 vol, p. 325.

59. Par exemple, Gérard Claude travaille sur un milieu marqué par une dominante sectorielle importante. En effet, les Italiens de Provence s'installent majoritairement dans les campagnes. De plus, l'immigration italienne dans cette région prend un caractère beaucoup plus massif que dans le Maine-et-Loire. 
Finalement, la question de l'intégration ne s'est même pas posée pour ces Italiens. La ville de Saumur a servi en premier lieu de refuge aux exilés politiques romagnols. Par la suite, la création d'une petite implantation transalpine fait de cette sous-préfecture un creuset occasionnel pour ces étrangers. La lente sédimentation opérée leur permet de s'insérer naturellement dans la société d'accueil. Le critère de nationalité ${ }^{60}$ ne constitue pas un obstacle à l'installation des transalpins. Par conséquent, la formation de cette micro-colonie italienne et l'intégration ${ }^{61}$ de ses membres dans le milieu récepteur se sont effectuées sans heurts, d'une façon très libérale durant une période et dans une ville où le " problème étranger " ne se pose pas.

60. Les naturalisations sont très peu nombreuses. Ainsi, seul Louis Liverani a demandé et obtenu la nationalité française. L'absence de telle pratique pourrait être un indice supplémentaire d'une bonne intégration. En effet, une insertion réussie est souvent le résultat d'une acceptation de la part du milieu d'accueil et rend moins nécessaire le recours à la naturalisation

61. Nous n'avons pas parlé des mariages mixtes car ils sont délicats à interpréter. En effet, aucune union entre Italiens n'a pu être repérée. Est-ce là le signe d'une volonté farouche d'insertion? Rien n'est moins sûr puisque l'étroitesse du marché matrimonial transalpin imposait peut-être des mariages franco-italiens. 


\section{RESUME}

Une cinquantaine de réfugiés politiques provenant des environs de Rimini, en Emilie-Romagne ont été conduits à Saumur à la fin de l'année 1845. La prise en charge de ces républicains par les autorités et la population dans une conjoncture économique déprimée va poser de nombreux problèmes. Toutefois, l'accueil de ces hommes, jusqu'au départ de la majorité d'entre eux en 1846, sera sans faille. Les quelques exilés restés dans la ville vont être à l'origine de la formation d'une micro-colonie italienne (entre 20 à 30 membres) à Saumur. L'intégration économique de ces transalpins dans un milieu marqué une présence étrangère anecdotique est relativement modeste. Pourtant, l'ascension sociale de certains Italiens montre que cette société d'accueil pouvait être un terreau favorable pour une intégration rapide et un enrichissement important. La carrière politique d'un des membres de cette communauté, Angelo Bolognesi, prouve également l'ouverture du milieu récepteur. Enfin, l'existence d'un entre-soi italien durant cet exil constitue un phénomène remarquable puisque la faiblesse numérique du contingent et son éparpillement au sein de la ville ne favorise pas, a priori, le maintien de cette sociabilité migratoire.

\section{ABSTRACT}

Fifty or so political refugees from the areas surrounding Rimini, in EmilieRomagne, were taken to Saumur at the end of 1845. The population and the authorities found it difficult to take care of these republicans due to the economic depression at the time. However, these men were well taken care of until the last of them left in 1846. A few of them stayed in the town and were the founders of an Italian micro-colony (between 20 and 30 members) in Saumur. The economic integration of these immigrants from the other side of the Alps was relatively modest in such a community where few foreigners could be found. However, the rapid social ascension of some of these Italians was proof that this host society could offer a rapid integration and important opportunities to gain wealth. The political career of one of the members of this community, Angelo Bolognesi, also shows the openness of this society. Finally, the existence of an identity during this exile is remarkable given the small number of men and their scattered geographical locations within the town. 



\section{II \\ Micro-immigrations économiques au XIX ${ }^{\mathrm{e}}$ et au début du $\mathrm{Xx}^{\mathrm{e}}$ siècle}


